\title{
Community Composition and Distribution Pattern of Herbaceous Flora in Holi Area of District Chamba in Himachal Pradesh
}

\author{
Kehar Singh Thakur*, Munesh Kumar, Rajan Bawa, Anita Kumari and Anurag Sharma
}

Krishi Vigyan Kendra Chamba, H.P. (176 314), India

\author{
Corresponding Author \\ Kehar Singh Thakur \\ e-mail: thakur.kehar.kst@gmail.com
}

\author{
Article History \\ Article ID: IJEP0354 \\ Received in $29^{\text {th }}$ December, 2019 \\ Received in revised form $14^{\text {th }}$ January, 2020 \\ Accepted in final form $23^{\text {rd }}$ January, 2020
}

\begin{abstract}
The present study was carried out in Holi Forest Range of district Chamba, which is the north-west district of Himachal Pradesh and is located between latitude $32^{\circ} 17^{\prime} 412^{\prime \prime}$ to $32^{\circ} 26^{\prime} 541^{\prime \prime} \mathrm{N}$ and longitude $76^{\circ} 31^{\prime} 504^{\prime \prime}$ to $76^{\circ} 35^{\prime} 385^{\prime \prime}$. The territory is completely mountainous with altitude varying from about 2,000 feet $(610 \mathrm{~m})$ to about 21,000 feet $(6,400 \mathrm{~m})$ above the mean sea level. The quantitative information of herbs was collected from different sites i.e., Deol, Kut, Dal, Lahaud Dhar. Frequency (\%), Density (plants $\left.\mathrm{m}^{-2}\right)$, Basal Area $\left(\mathrm{cm}^{2}\right)$, Importance Value Index (IVI) and A/F ratio of plant species at different sites were recorded. Poa alpina was found to have highest frequency (90\%) closely followed by Jurinea dolomiaea (80\%) and Biebersteinia odora has the lowest frequency of $10 \%$. Poa alpina has the highest density (60.6 plants $\mathrm{m}^{-2}$ ) at Dal followed by Gentiana kurrooa (35.8 plants $\left.\mathrm{m}^{-2}\right)$. Moschela esculenta was found to have highest basal area $\left(1.234 \mathrm{~cm}^{2}\right)$ at Kut. Viola serpens was the dominant species with highest value of IVI (78.77) closely followed by Poa alpina (65.91), Gentiana kurrooa (65.37) and Jurinea dolomiaea (65.36). Most of the species were distributed randomly followed by contagious pattern of distribution and least species were reported for regular distribution pattern.
\end{abstract}

Keywords: A/f ratio, basal area, herbaceous flora, importance value index

\section{Introduction}

Chamba district of Himachal Pradesh is considered as one of the richest area of traditional and potential medicinal wealth. The district is bounded by Kangra district of Himachal Pradesh and Gurdaspur district in the south of Punjab, Jammu and Kashmir in the north and Lahaul Spiti in the west. The district has two tribal regions, viz., Pangi and Bharmour. Bharmour is situated in the west of this district, whereas Pangi Valley is situated in the north. The vegetation of the district Chamba varies considerably, chiefly owing to elevation and rainfall variations.

The unprecedented rate of species extinction in recent times projects about quarter of the global species to be lost or threatened by the middle of $21^{\text {st }}$ century (Raven, 1990). With growing global concern for species endangerment, especially over the past two decades, the term biodiversity has encouraged conservationists to look for causes and consequences of species extinction and finding ways for their conservation. Human activities and unsustainable harvesting in the wild have been identified as one of the biggest causes of reported phenomenal loss of species (Lewin, 1986; Wilson, 1988). The recent IUCN Red List sampled 91\% plant species as threatened due to habitat loss and degradation (Hilton, 2000).
India is ranked at sixth place for having the largest number of threatened plant species in the above IUCN Red List. It is well understood to extinction and by understanding the processes that contribute to their rarity, future loss of diversity may be deferred or reduced (Flather et al., 1994).

With growing awareness of the people towards the use of herbal medicine during mid 1980s to the 1990s, about 233 major pharmaceutical companies globally became involved in screening of plants for new leads (Aryal, 1993). Nearly 2,500 wild plant species are reported in use for medicinal purpose in Indian subcontinent, of which, possibly about 300 taxa are used in 8,000 licensed pharmaceuticals in India (Ahmad, 1993). In recent years, India has emerged as one of the biggest suppliers of raw material (Holley and Cherla, 1998) ranking second amongst 12 world leading exporter countries (Lange, 1997). Collection of medicinal herbs as Minor Forest Produce (MFP) under forest law as traditional rights in designated forest land (Anderson, 1886) has been an important source of the native's income in Himachal Himalaya (Dobriyal et al., 1997; Tandon, 1997; Badola, 1998, 2002).

A rough estimate and secondary sources suggest availability of about 1,000 to 1,200 medicinal plant species in Himachal Himalayas (Gupta, 1964, 1971; Gaur and Singh, 1993; 
Chauhan, 1999; Badola, 2001). As per the habitat type, these share $18 \%$ trees, $21 \%$ shrubs, $55 \%$ herbs in composition, which coincides more or less with that of Indian Himalayan Region, having $23 \%, 22 \%$ and $58 \%$ species of trees, shrubs and herbs, respectively (Samant et al., 1998).

Kumar et al. (2010) carried out a study of ecological status of ethno medicinal plants in the Garhwal Himalaya. The northern part of India harbours a great diversity of medicinal plants due to its distinct geography and ecological marginal conditions. They reported total of 57 species, including 14 trees, 10 shrubs and 33 herbs. Regular and random distribution pattern of species reflect the higher biotic pressure in terms of grazing and lopping in natural forest stands (Kumar et al., 2010). Shameem and Kangroo (2011) carried out a study in Dachigam National Park, Kashmir Himalaya, India reported that most of the species in their study were distributed contagiously. The studies carried out by Shadangi and Nath (2005) also gave similar findings of contagious distribution pattern of species.

In Study area, it has been reported that a number of species like Angelica gluaca, Picrorhiza kurroo, Jurinea dolomiaea and Podophyllum hexandrum are under the threat of extension (Dinanath, 2007). This calls upon the taxonomists and economic botanists to undertake systematic studies on the existing flora to identify and inventoried the medicinal and aromatic plant species enabling the scientists, planners and administrators to initiate effective steps for their conservation and sustainable utilization, otherwise the area may lose some of these species forever. Furthermore, tribal people residing in the study area, since thousands of years have been interacting with the flora and have evolved their own traditional healing methods, relying heavily on local medicinal plant resources (Karki and Willians, 1999). There is no proper record available regarding the community composition and distribution of the medicinal plant diversity of Holi area. Keeping these factors in view, the present study was carried out with the objective to study community composition and distribution pattern of herbaceous flora.

\section{Materials and Methods}

The present study was carried out in Holi Forest Range of district Chamba, which is located between latitude $32^{\circ} 17^{\prime} 412^{\prime \prime}$ to $32^{\circ} 26^{\prime} 541^{\prime \prime} \mathrm{N}$ and longitude $76^{\circ} 31^{\prime} 504^{\prime \prime}$ to $76^{\circ} 35^{\prime} 385^{\prime \prime} \mathrm{E}$. Extensive field survey of the selected areas of Holi starting from the lower elevation at Deol (2,300-2800 m), Kut (2,800$3300 \mathrm{~m})$, Dal (3,300-3800 m) and Lahaud Dhar (3,800 m and above) under Holi Forest Range was carried out (Table 1 and Figure 1).

For the vegetation analysis of herbaceous layer, a total of 160 plots of $1 \times 1 \mathrm{~m}$ size quadrate laid out randomly in the study area. Species richness was simply taken as a count of number of species present in that forest type. The vegetation data were quantitatively analyzed for density, frequency and abundance (Curtis and McIntosh, 1950). Importance Value

\begin{tabular}{|c|c|c|c|c|}
\hline \multirow{2}{*}{$\begin{array}{l}\text { Forest } \\
\text { range }\end{array}$} & \multirow[t]{2}{*}{ Site } & \multirow{2}{*}{$\begin{array}{l}\text { Altitude } \\
\text { (m) }\end{array}$} & \multicolumn{2}{|c|}{ Coordinates } \\
\hline & & & Longitude & Latitude \\
\hline \multirow[t]{4}{*}{ Holi } & Deol & $\begin{array}{l}2300- \\
2800\end{array}$ & $32018^{\prime \prime} 079^{\prime} \mathrm{N}$ & $076035^{\prime 2} 262^{\prime} \mathrm{E}$ \\
\hline & Kut & $\begin{array}{l}2800- \\
3300\end{array}$ & $32017 " 488^{\prime N}$ & $076034 " 870 ’ E$ \\
\hline & Dal & $\begin{array}{l}3300- \\
3800\end{array}$ & $32017 " 412^{\prime} \mathrm{N}$ & 076032 "342'E \\
\hline & $\begin{array}{l}\text { Lahaud } \\
\text { Dhar }\end{array}$ & $\begin{array}{c}3800 \text { and } \\
\text { above }\end{array}$ & $32017 " 208^{\prime} \mathrm{N}$ & $076032 " 002$ 'E \\
\hline
\end{tabular}

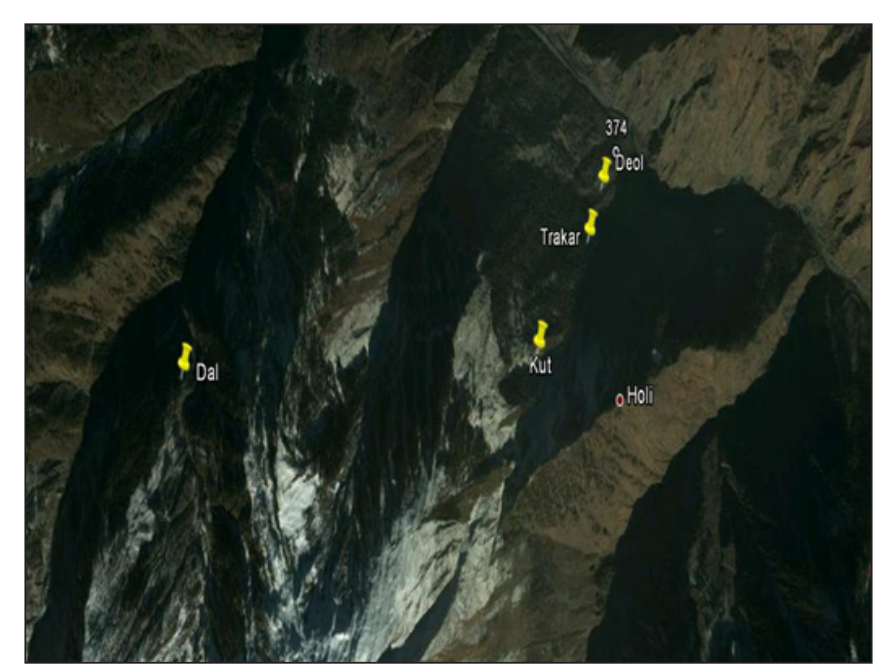

Figure 1: Sites of study area (Holi)

Index (IVI) was calculated using the sum of relative frequency, relative density and relative dominance (Phillips, 1959). The quantitative analysis for different parameters was calculated as follows:

Frequency (\%)

Frequency indicates the number of sampling units in which a given species occur. Percent frequency was calculated as follows:

Frequency $(\%)=($ No. of sampling units in which species occurred $\div$ Total number of sampling units studied) $\times 100$

\section{Density}

It represents the numerical strength of species in a community. Density was calculated as follows:

Density $=($ Total number of indivuduals of a species in all sampling units $\div$ Total number os sampling units studies) $\times 100$

\section{Abundance}

Abundance is analyzed to get an idea of distribution pattern of the species.

Abundance $=$ (Total number of individuals of a species in all sampling units $\div$ Total number of sampling units in which species occurred) 


\section{Basal area}

Basal area is the area of ground actually penetrated by the stems, and is readily seen when the leaves and stems are clipped at the ground surface. Basal area of herbs was measured at the ground level which is calculated as:

Basal area $=\pi r^{2}$

Importance value Index (IVI)

The IVI which is an integrated measure of the relative frequency, relative density and relative basal area, was calculated from the basic data for each species of herbs (Phillips, 1959) as:

Importance value index=Relative frequency+relative density+relative dominance

The relative values of frequency, density and basal area was calculated as follows:

Relative frequency $=($ Frequency of individual speices $\div$ Frequency of all speices $) \times 100$

Relative density $=$ (Density of individual speices $\div$ Density of all speices) $\times 100$

Relative dominance $=($ Basal area of individual speices $\div$ Basal area of all speices) $\times 100$

\section{Abundance to frequency ratio}

Abundance to Frequency ratio ( $A / F$ ratio) for different species was determined for eliciting the distribution pattern. The distribution pattern of species is considered regular if ratio is $<0.025$, random if ratio between 0.025-0.05 and contagious if ratio $>0.05$ (Curtis and Cottam, 1956; Whitford, 1949) as:

$\mathrm{A} / \mathrm{F}$ ratio $=$ Abundance $\div$ Frequency

\section{Results and Discussion}

Frequency, density, basal area and importance value index of plant species at Deol are presented in Table 2. It has been observed that Diplazium esculantum has the highest frequency $(70 \%)$ closely followed by Lecanthus peduncularis and Morchella esculenta (60\% each) whereas, Ainsliaea aptera has the lowest frequency (10\%). Nasturtium officinale has the highest density (10.0 plants $\mathrm{m}^{-2}$ ) followed by Fagopyrum esculentum (6.2 plants $\mathrm{m}^{-2}$ ) whereas, Ainsliaea aptera has the lowest density $\left(0.2\right.$ plants $\left.\mathrm{m}^{-2}\right)$. Verbascum cylindericum was found to have highest basal area $\left(0.622 \mathrm{~cm}^{2}\right)$ followed by Artemisia vulgaris $\left(0.520 \mathrm{~cm}^{2}\right)$ whereas, Chrysopogon gryllus and Cynodon dactylon has the lowest basal area $\left(0.002 \mathrm{~cm}^{2}\right)$. Morchella esculenta has the highest value of IVI (16.58) followed by Diplazium esculantum (13.77) whereas, Ainsliaea aptera has the lowest value of IVI (0.85). Thus, depicting that Morchella esculenta was the dominant species, Diplazium esculantum codominant and Ainsliaea aptera the associated species. At Deol, the highest numbers of species
Table 2: Frequency, density, basal area and importance value index of plant species at Deol

\begin{tabular}{|c|c|c|c|c|c|c|}
\hline $\begin{array}{l}\text { Sr. } \\
\text { No. }\end{array}$ & Species & $\begin{array}{c}\text { Fre- } \\
\text { quen- } \\
\text { cy } \\
(\%)\end{array}$ & $\begin{array}{c}\text { Den- } \\
\text { sity } \\
\text { (plants } \\
\mathrm{m}^{-2} \text { ) }\end{array}$ & $\begin{array}{l}\text { Basal } \\
\text { Area } \\
\left(\mathrm{cm}^{2}\right)\end{array}$ & IVI & $\begin{array}{l}\mathrm{A} / \mathrm{F} \\
\text { ratio }\end{array}$ \\
\hline 1 & $\begin{array}{l}\text { Ainsliaea ap- } \\
\text { tera }\end{array}$ & 10 & 0.2 & 0.012 & 0.85 & 0.020 \\
\hline 2 & $\begin{array}{l}\text { Amaranthus } \\
\text { paniculatus }\end{array}$ & 20 & 0.9 & 0.080 & 2.61 & 0.005 \\
\hline 3 & $\begin{array}{l}\text { Arisaema fla- } \\
\text { vum }\end{array}$ & 20 & 2.0 & 0.311 & 5.33 & 0.100 \\
\hline 4 & $\begin{array}{l}\text { Arte misia } \\
\text { brevifolia }\end{array}$ & 20 & 0.5 & 0.487 & 5.01 & 0.025 \\
\hline 5 & $\begin{array}{l}\text { Artemisia vul- } \\
\text { garis }\end{array}$ & 40 & 1.0 & 0.520 & 6.87 & 0.025 \\
\hline 6 & $\begin{array}{l}\text { Arundinella } \\
\text { nepalensis }\end{array}$ & 20 & 0.4 & 0.319 & 3.80 & 0.020 \\
\hline 7 & $\begin{array}{l}\text { Aster diplo- } \\
\text { stephioides }\end{array}$ & 20 & 0.5 & 0.311 & 3.80 & 0.025 \\
\hline 8 & $\begin{array}{l}\text { Aster him- } \\
\text { alaicus }\end{array}$ & 20 & 0.4 & 0.317 & 3.74 & 0.020 \\
\hline 9 & $\begin{array}{l}\text { Bistorta am- } \\
\text { plexicaulis }\end{array}$ & 30 & 0.8 & 0.020 & 2.66 & 0.027 \\
\hline 10 & $\begin{array}{l}\text { Bistorta im- } \\
\text { pleriscor }\end{array}$ & 40 & 1.2 & 0.026 & 3.67 & 0.030 \\
\hline 11 & $\begin{array}{l}\text { Cannabis sa- } \\
\text { tiva }\end{array}$ & 30 & 1.1 & 0.518 & 6.40 & 0.037 \\
\hline 12 & $\begin{array}{l}\text { Capllipedium } \\
\text { parviflorum }\end{array}$ & 20 & 0.8 & 0.499 & 5.41 & 0.040 \\
\hline 13 & Carum carvi & 30 & 3.1 & 0.013 & 4.94 & 0.103 \\
\hline 14 & $\begin{array}{l}\text { Chaerophyl- } \\
\text { lum reflexum }\end{array}$ & 30 & 0.6 & 0.011 & 2.39 & 0.020 \\
\hline 15 & $\begin{array}{l}\text { Chenopodium } \\
\text { album }\end{array}$ & 20 & 0.8 & 0.468 & 5.19 & 0.040 \\
\hline 16 & $\begin{array}{l}\text { Chrysopogon } \\
\text { gryllus }\end{array}$ & 40 & 2.6 & 0.002 & 4.92 & 0.065 \\
\hline 17 & $\begin{array}{l}\text { Coriandrum } \\
\text { sativum }\end{array}$ & 20 & 1.3 & 0.015 & 2.57 & 0.065 \\
\hline 18 & $\begin{array}{l}\text { Cyathula cap- } \\
\text { itata }\end{array}$ & 20 & 0.4 & 0.485 & 4.90 & 0.020 \\
\hline 19 & $\begin{array}{l}\text { Cynodon dac- } \\
\text { tylon }\end{array}$ & 30 & 1.1 & 0.002 & 2.83 & 0.037 \\
\hline 20 & $\begin{array}{l}\text { Diplazium es- } \\
\text { culantum }\end{array}$ & 70 & 6.1 & 0.519 & 13.77 & 0.087 \\
\hline 21 & $\begin{array}{l}\text { Diplazium } \\
\text { polypodioides }\end{array}$ & 30 & 1.2 & 0.502 & 6.39 & 0.040 \\
\hline
\end{tabular}

Table 2: Continue... 


\begin{tabular}{|c|c|c|c|c|c|c|c|c|c|c|c|c|c|}
\hline $\begin{array}{l}\text { Sr. } \\
\text { No. }\end{array}$ & Species & $\begin{array}{c}\text { Fre- } \\
\text { quen- } \\
\text { cy } \\
(\%)\end{array}$ & $\begin{array}{l}\text { Den- } \\
\text { sity } \\
\text { (plants } \\
\mathrm{m}^{-2} \text { ) }\end{array}$ & $\begin{array}{l}\text { Basal } \\
\text { Area } \\
\left(\mathrm{cm}^{2}\right)\end{array}$ & IVI & $\begin{array}{l}\mathrm{A} / \mathrm{F} \\
\text { ratio }\end{array}$ & $\begin{array}{l}\text { Sr. } \\
\text { No. }\end{array}$ & Species & $\begin{array}{c}\text { Fre- } \\
\text { quen- } \\
\text { cy } \\
(\%)\end{array}$ & $\begin{array}{c}\text { Den- } \\
\text { sity } \\
\text { (plants } \\
\mathrm{m}^{-2} \text { ) }\end{array}$ & $\begin{array}{l}\text { Basal } \\
\text { Area } \\
\left(\mathrm{cm}^{2}\right)\end{array}$ & IVI & $\begin{array}{l}\mathrm{A} / \mathrm{F} \\
\text { ratio }\end{array}$ \\
\hline 22 & $\begin{array}{l}\text { Dryopteris ni- } \\
\text { gropalaceum }\end{array}$ & 50 & 0.7 & 0.011 & 3.62 & 0.014 & 41 & $\begin{array}{l}\text { Ranunculus } \\
\text { hirtellus }\end{array}$ & 20 & 0.8 & 0.022 & 2.11 & 0.040 \\
\hline 23 & $\begin{array}{l}\text { Equesetum } \\
\text { arvensis }\end{array}$ & 20 & 0.8 & 0.469 & 5.20 & 0.020 & 42 & $\begin{array}{l}\text { Rumex nepal- } \\
\text { ensis }\end{array}$ & 30 & 2.0 & 0.317 & 5.92 & 0.067 \\
\hline 24 & $\begin{array}{l}\text { Fagopyrum } \\
\text { dibotrys }\end{array}$ & 20 & 0.6 & 0.492 & 5.15 & 0.030 & 43 & $\begin{array}{l}\text { Sanicula eu- } \\
\text { ropaea }\end{array}$ & 40 & 3.6 & 0.013 & 6.03 & 0.090 \\
\hline 25 & $\begin{array}{l}\text { Fagopyrum } \\
\text { esculentum }\end{array}$ & 40 & 6.2 & 0.485 & 11.93 & 0.155 & 44 & $\begin{array}{l}\text { Senecio chry- } \\
\text { senthemoides }\end{array}$ & 50 & 3.7 & 0.015 & 6.71 & 0.074 \\
\hline 26 & $\begin{array}{l}\text { Foeniculum } \\
\text { vulgare }\end{array}$ & 20 & 0.3 & 0.312 & 3.61 & 0.015 & 45 & Siegesbeckia & 20 & 0.6 & 0.020 & 1.89 & 0.030 \\
\hline 27 & $\begin{array}{l}\text { Fragaria ves- } \\
\mathrm{ca}\end{array}$ & 40 & 1.4 & 0.300 & 5.77 & 0.035 & 46 & Silene cucu- & 20 & 0.3 & 0.018 & 1.57 & 0.015 \\
\hline 28 & $\begin{array}{l}\text { Galium triflo- } \\
\text { rum }\end{array}$ & 20 & 1.1 & 0.435 & 5.27 & 0.055 & 47 & Silene edge- & 30 & 0.5 & 0.021 & 2.36 & 0.017 \\
\hline 29 & $\begin{array}{l}\text { Geranium } \\
\text { robertianum }\end{array}$ & 30 & 0.8 & 0.238 & 4.17 & 0.027 & 48 & $\begin{array}{l}\text { worthii } \\
\text { Silene viscose }\end{array}$ & 20 & 0.4 & 0.026 & 1.73 & 0.020 \\
\hline 30 & $\begin{array}{l}\text { Impetiens } \\
\text { balsamina }\end{array}$ & 20 & 0.4 & 0.219 & 3.07 & 0.020 & 49 & $\begin{array}{l}\text { Stellaria } \\
\text { aquatic }\end{array}$ & 30 & 0.3 & 0.032 & 2.23 & 0.010 \\
\hline 31 & $\begin{array}{l}\text { Impetiens } \\
\text { laxiflora }\end{array}$ & 20 & 0.5 & 0.208 & 3.09 & 0.025 & 50 & $\begin{array}{l}\text { Stellaria cris- } \\
\text { pate }\end{array}$ & 20 & 0.7 & 0.041 & 2.13 & 0.350 \\
\hline 32 & $\begin{array}{l}\text { Inula cuspi- } \\
\text { date }\end{array}$ & 30 & 1.3 & 0.478 & 6.33 & 0.043 & 51 & $\begin{array}{l}\text { Taraxacum } \\
\text { officinale }\end{array}$ & 30 & 0.8 & 0.018 & 2.64 & 0.027 \\
\hline 33 & $\begin{array}{l}\text { Lecanthus pe- } \\
\text { duncularis }\end{array}$ & 60 & 5.4 & 0.499 & 12.35 & 0.090 & 52 & $\begin{array}{l}\text { The me d a } \\
\text { anathera }\end{array}$ & 30 & 1.2 & 0.007 & 2.97 & 0.040 \\
\hline 34 & $\begin{array}{l}\text { Malva gul- } \\
\text { sausan }\end{array}$ & 20 & 0.6 & 0.058 & 2.15 & 0.030 & 53 & $\begin{array}{l}\text { Trifolium re- } \\
\text { pense }\end{array}$ & 20 & 2.7 & 0.012 & 3.97 & 0.135 \\
\hline 35 & Melilotus of- & 30 & 0.8 & 0.034 & 2.76 & 0.027 & 54 & Tulip stilata & 20 & 0.3 & 0.047 & 1.78 & 0.015 \\
\hline & & & & & & & 55 & Urtica dioca & 20 & 1.5 & 0.312 & 4.83 & 0.075 \\
\hline 36 & $\begin{array}{l}\text { Mentha lon- } \\
\text { gifolia }\end{array}$ & 40 & 3.4 & 0.488 & 9.11 & 0.085 & 56 & $\begin{array}{l}\text { Urtica parvi- } \\
\text { flora }\end{array}$ & 30 & 1.3 & 0.330 & 5.31 & 0.043 \\
\hline 37 & $\begin{array}{l}\text { Mentha viri- } \\
\text { dis }\end{array}$ & 30 & 6.0 & 0.348 & 10.21 & 0.200 & 57 & $\begin{array}{l}\text { Valeriana } \\
\text { jatamansi }\end{array}$ & 40 & 1.8 & 0.401 & 6.87 & 0.045 \\
\hline 38 & $\begin{array}{l}\text { Microstegium } \\
\text { nudum }\end{array}$ & 30 & 0.8 & 0.011 & 2.60 & 0.027 & 58 & Verbascum & 20 & 0.3 & 0.622 & 5.75 & 0.015 \\
\hline 39 & $\begin{array}{l}\text { Morchella es- } \\
\text { culenta }\end{array}$ & 60 & 2.8 & 1.493 & 16.58 & 0.047 & 59 & $\begin{array}{l}\text { cyinaericum } \\
\text { Viola pilosa }\end{array}$ & 30 & 3.4 & 0.075 & 5.76 & 0.113 \\
\hline 40 & $\begin{array}{l}\text { Nasturtium } \\
\text { officinale }\end{array}$ & 40 & 10.0 & 0.012 & 12.53 & 0.250 & 60 & $\begin{array}{l}\text { Viola serpens } \\
\text { Total }\end{array}$ & 40 & 1.0 & 0.070 & 3.89 & 0.025 \\
\hline
\end{tabular}

were distributed randomly followed by contagious pattern of distribution and lowest species were distributed regularly.

Frequency, density, basal area and importance value index at Kut, are shown in Table 3. Viola serpens was found to have highest frequency $(70 \%)$ closely followed by Valeriana jatamensii (60\%) whereas, Artica parviflora has the lowest frequency (10\%). Viola serpens was found to have highest density ( 85.5 plants $\mathrm{m}^{-2}$ ) followed by Valeriana jatamensii (5.8 plants $\mathrm{m}^{-2}$ ) whereas, Artica parviflora has the lowest density (0.5 plants $\left.\mathrm{m}^{-2}\right)$. Moschela esculenta was found to have highest basal area $\left(1.234 \mathrm{~cm}^{2}\right)$ followed by Gentiana karrooa $(0.656$ $\mathrm{cm}^{2}$ ) whereas, Agrostis species has the lowest basal area 


\begin{tabular}{|c|c|c|c|c|c|c|c|c|c|c|c|c|c|}
\hline $\begin{array}{l}\text { Sr. } \\
\text { No. }\end{array}$ & Species & $\begin{array}{c}\text { Fre- } \\
\text { quen- } \\
\text { cy } \\
(\%)\end{array}$ & $\begin{array}{c}\text { Den- } \\
\text { sity } \\
\text { (plants } \\
\mathrm{m}^{-2} \text { ) }\end{array}$ & $\begin{array}{l}\text { Basal } \\
\text { Area } \\
\left(\mathrm{cm}^{2}\right)\end{array}$ & IVI & $\begin{array}{l}\mathrm{A} / \mathrm{F} \\
\text { ratio }\end{array}$ & $\begin{array}{l}\text { Sr. } \\
\text { No. }\end{array}$ & Species & $\begin{array}{c}\text { Fre- } \\
\text { quen- } \\
\text { cy } \\
(\%)\end{array}$ & $\begin{array}{l}\text { Den- } \\
\text { sity } \\
\text { (plants } \\
\mathrm{m}^{-2} \text { ) }\end{array}$ & $\begin{array}{l}\text { Basal } \\
\text { area } \\
\left(\mathrm{cm}^{2}\right)\end{array}$ & IVI & $\begin{array}{l}\mathrm{A} / \mathrm{F} \\
\text { ratio }\end{array}$ \\
\hline 1 & $\begin{array}{l}\text { Achillea mille- } \\
\text { folium }\end{array}$ & 20 & 0.6 & 0.020 & 2.21 & 0.030 & 23 & $\begin{array}{l}\text { Hackelia un- } \\
\text { cinata }\end{array}$ & 20 & 0.9 & 0.054 & 3.81 & 0.045 \\
\hline 2 & $\begin{array}{l}\text { Agrimonia } \\
\text { pilosa }\end{array}$ & 20 & 0.8 & 0.060 & 3.23 & 0.040 & 24 & $\begin{array}{l}\text { Heracleum } \\
\text { candicans }\end{array}$ & 20 & 0.7 & 0.032 & 2.53 & 0.035 \\
\hline \multirow[t]{2}{*}{3} & \multirow{2}{*}{$\begin{array}{l}\text { Agrostis spe- } \\
\text { cies }\end{array}$} & \multirow[t]{2}{*}{20} & \multirow[t]{2}{*}{1.2} & \multirow[t]{2}{*}{0.002} & \multirow[t]{2}{*}{2.02} & \multirow[t]{2}{*}{0.060} & 25 & Inula cappa & 30 & 1.2 & 0.087 & 4.77 & 0.040 \\
\hline & & & & & & & 26 & Inula hookeri & 20 & 0.8 & 0.069 & 3.44 & 0.040 \\
\hline 4 & $\begin{array}{l}\text { Ajuga brac- } \\
\text { teosa }\end{array}$ & 20 & 1.5 & 0.052 & 3.31 & 0.075 & 27 & $\begin{array}{l}\text { Leontopodi- } \\
\text { um stracheyi }\end{array}$ & 30 & 1.5 & 0.022 & 3.37 & 0.050 \\
\hline 5 & $\begin{array}{l}\text { Angelica } \\
\text { glauca }\end{array}$ & 30 & 2.0 & 0.064 & 4.55 & 0.067 & 28 & $\begin{array}{l}\text { Moschela } \\
\text { esculenta }\end{array}$ & 50 & 4.8 & 1.234 & 34.58 & 0.096 \\
\hline 6 & $\begin{array}{l}\text { Artemisia rox- } \\
\text { burghii }\end{array}$ & 40 & 4.5 & 0.279 & 11.33 & 0.113 & 29 & $\begin{array}{l}\text { Origanum } \\
\text { bulgar }\end{array}$ & 20 & 0.9 & 0.056 & 3.17 & 0.045 \\
\hline 7 & $\begin{array}{l}\text { Artica parvi- } \\
\text { flora }\end{array}$ & 10 & 0.5 & 0.023 & 1.49 & 0.051 & 30 & $\begin{array}{l}\text { Plantago hi- } \\
\text { malacia }\end{array}$ & 20 & 1.2 & 0.021 & 2.46 & 0.060 \\
\hline 8 & $\begin{array}{l}\text { Artemisia } a b- \\
\text { sinthium }\end{array}$ & 30 & 3.5 & 0.200 & 8.33 & 0.117 & 31 & $\begin{array}{l}\text { Plantago } \\
\text { lanceolata }\end{array}$ & 30 & 0.8 & 0.009 & 2.79 & 0.027 \\
\hline 9 & $\begin{array}{l}\text { Bistorta am- } \\
\text { plexicaulis }\end{array}$ & 20 & 0.8 & 0.012 & 2.10 & 0.040 & 32 & $\begin{array}{l}\text { Pleurosper- } \\
\text { mum bruno- }\end{array}$ & 30 & 1.3 & 0.011 & 3.03 & 0.043 \\
\hline 10 & Bupleurum & 20 & 0.6 & 0.006 & 1.88 & 0.030 & & nis & & & & & \\
\hline 11 & $\begin{array}{l}\text { falcatum } \\
\text { Bupleurum }\end{array}$ & 20 & 0.7 & 0.013 & 2.08 & 0.035 & 33 & $\begin{array}{l}\text { Potentilla at- } \\
\text { rosanguinea }\end{array}$ & 20 & 1.2 & 0.008 & 2.16 & 0.060 \\
\hline 12 & Cassiope fas- & 30 & 1.1 & 0.021 & 3.18 & 0.037 & 34 & $\begin{array}{l}\text { Potentilla } \\
\text { neplensis }\end{array}$ & 30 & 0.8 & 0.076 & 4.36 & 0.027 \\
\hline 13 & Chaerophyl- & 20 & 1.3 & 0.030 & 2.02 & 0.065 & 35 & $\begin{array}{l}\text { Prunela vul- } \\
\text { garis }\end{array}$ & 20 & 0.9 & 0.022 & 2.38 & 0.045 \\
\hline 14 & $\begin{array}{l}\text { lum villosum } \\
\text { Chrysopogon }\end{array}$ & 50 & 3.8 & 0.005 & 5.38 & 0.076 & 36 & $\begin{array}{l}\text { Ranuniculus } \\
\text { arvensis }\end{array}$ & 30 & 0.6 & 0.012 & 2.78 & 0.020 \\
\hline 15 & $\begin{array}{l}\text { gryllus } \\
\text { Corydalis cra- }\end{array}$ & 30 & 2.1 & 0.043 & 4.10 & 0.070 & 37 & $\begin{array}{l}\text { Ranuniculus } \\
\text { hirtellus }\end{array}$ & 20 & 0.8 & 0.015 & 2.17 & 0.040 \\
\hline 16 & Cotoneaster & 20 & 0.9 & 0.034 & 2.66 & 0.045 & 38 & $\begin{array}{l}\text { Ranuniculus } \\
\text { laetus }\end{array}$ & 20 & 0.6 & 0.018 & 2.16 & 0.030 \\
\hline 17 & Cremanthodi- & 20 & 0.8 & 0.065 & 3.34 & 0.040 & 39 & $\begin{array}{l}\text { Saussurea } \\
\text { lappa }\end{array}$ & 40 & 1.3 & 0.020 & 3.99 & 0.0325 \\
\hline 18 & Cynoglossum & 20 & 0.6 & 0.029 & 2.42 & 0.030 & 40 & $\begin{array}{l}\text { Selinium } \\
\text { vaginatum }\end{array}$ & 20 & 1.1 & 0.006 & 2.07 & 0.055 \\
\hline 19 & Fragaria & 50 & 3.6 & 0.017 & 5.58 & 0.072 & 41 & $\begin{array}{l}\text { Thymus } \\
\text { hexandrum }\end{array}$ & 20 & 1.4 & 0.100 & 4.39 & 0.070 \\
\hline 20 & $\begin{array}{l}\text { vesca } \\
\text { Gentiana kar- }\end{array}$ & 40 & 1.2 & 0.656 & 18.85 & 0.030 & 42 & $\begin{array}{l}\text { Thymus ser- } \\
\text { phyllum }\end{array}$ & 30 & 1.2 & 0.011 & 2.99 & 0.040 \\
\hline 21 & Geum elatum & 30 & 0.7 & 0.033 & 3.31 & 0.023 & 43 & $\begin{array}{l}\text { Thymus vul- } \\
\text { garus }\end{array}$ & 20 & 0.8 & 0.009 & 2.03 & 0.040 \\
\hline 22 & $\begin{array}{l}\text { Gynura } \\
\text { cusimbua }\end{array}$ & 30 & 0.8 & 0.025 & 3.17 & 0.040 & 44 & $\begin{array}{l}\text { Urginia in- } \\
\text { dica }\end{array}$ & 20 & 1.1 & 0.021 & 2.42 & 0.055 \\
\hline
\end{tabular}




\begin{tabular}{|c|c|c|c|c|c|c|}
\hline $\begin{array}{l}\text { Sr. } \\
\text { No. }\end{array}$ & Species & $\begin{array}{l}\text { Fre- } \\
\text { quen- } \\
\text { cy } \\
(\%)\end{array}$ & $\begin{array}{l}\text { Den- } \\
\text { sity } \\
\text { (plants } \\
\mathrm{m}^{-2} \text { ) }\end{array}$ & $\begin{array}{l}\text { Basal } \\
\text { area } \\
\left(\mathrm{cm}^{2}\right)\end{array}$ & IVI & $\begin{array}{l}\mathrm{A} / \mathrm{F} \\
\text { ratio }\end{array}$ \\
\hline 45 & $\begin{array}{l}\text { Valeriana } \\
\text { jatamansi }\end{array}$ & 60 & 5.8 & 0.212 & 11.77 & 0.097 \\
\hline 46 & $\begin{array}{l}\text { Verbascum } \\
\text { Thapsus }\end{array}$ & 20 & 1.3 & 0.433 & 12.16 & 0.065 \\
\hline 47 & $\begin{array}{l}\text { Vincetoxi- } \\
\text { cum hirun- } \\
\text { dinaria }\end{array}$ & 30 & 0.9 & 0.012 & 2.90 & 0.030 \\
\hline \multirow[t]{2}{*}{48} & $\begin{array}{l}\text { Viola ser- } \\
\text { pens }\end{array}$ & 70 & 85.5 & 0.008 & 78.77 & 1.220 \\
\hline & Total & & 153 & 4.267 & & \\
\hline
\end{tabular}

$\left(0.002 \mathrm{~cm}^{2}\right)$. Viola serpens was the dominant species with highest value of IVI (78.77) followed by Morchella esculenta (34.58) as the codominant and Artica parviflora the associated species with IVI value of 1.49. At Kut, the distribution pattern of most species was reported random followed by contagious and least species were distributed contagiously.

Frequency, density, basal area and importance value index at Dal, are shown in Table 4. Poa alpina was found to have highest frequency $(90 \%)$ closely followed by Jurinea dolomiaea (80\%). Poa alpina was found to have highest density (60.6 plants $\mathrm{m}^{-2}$ ) followed by Poa annua ( 35.5 plants $\mathrm{m}^{-2}$ ) whereas, Saussurea taraxacifolia has the lowest density (0.6 plants $\left.\mathrm{m}^{-2}\right)$. Jurinea dolomiaea has the highest basal area $\left(0.485 \mathrm{~cm}^{2}\right)$ followed by Rheum moorcroftiasana $\left(0.366 \mathrm{~cm}^{2}\right)$ whereas, Poa alpina has the lowest basal area $\left(0.004 \mathrm{~cm}^{2}\right)$. Poa alpina has the highest value of IVI (65.91) followed by Poa annua (42.14) whereas, Buplerium falcatum has the lowest value

\begin{tabular}{|c|c|c|c|c|c|c|}
\hline Sr. No. & Species & Frequency (\%) & Density (plants $\mathrm{m}^{-2}$ ) & Basal area $\left(\mathrm{cm}^{2}\right)$ & IVI & $\mathrm{A} / \mathrm{F}$ ratio \\
\hline 1 & Aconitum heterophyllum & 20 & 1.1 & 0.020 & 3.50 & 0.055 \\
\hline 2 & Anaphalis basua & 30 & 1.6 & 0.015 & 4.67 & 0.053 \\
\hline 3 & Anaphalis triplinervis & 20 & 0.8 & 0.110 & 6.77 & 0.040 \\
\hline 4 & Anaphalis contorta & 30 & 1.3 & 0.103 & 7.87 & 0.043 \\
\hline 5 & Angelica gulaca & 20 & 1.3 & 0.054 & 4.81 & 0.065 \\
\hline 6 & Biebersteinia odora & 10 & 0.5 & 0.065 & 3.78 & 0.051 \\
\hline 7 & Buplerium falcatum & 20 & 0.7 & 0.006 & 2.87 & 0.035 \\
\hline 8 & Buplerium logicaule & 20 & 0.8 & 0.010 & 3.04 & 0.040 \\
\hline 9 & Chrysopogon gryllus & 30 & 3.8 & 0.008 & 4.97 & 0.127 \\
\hline 10 & Corydalis flabellate & 40 & 2.6 & 0.032 & 6.79 & 0.065 \\
\hline 11 & Cynodon dactylon & 50 & 8.9 & 0.007 & 8.70 & 0.178 \\
\hline 12 & Geranium rotundifolium & 30 & 1.8 & 0.014 & 4.68 & 0.060 \\
\hline 13 & Geranium wallichiana & 40 & 2.8 & 0.020 & 6.40 & 0.070 \\
\hline 14 & Gerbera gossypina & 20 & 0.9 & 0.011 & 3.11 & 0.045 \\
\hline 15 & Juniperus cuminus & 20 & 1.6 & 0.322 & 14.88 & 0.080 \\
\hline 16 & Jurinea dolomiaea & 80 & 4.8 & 0.485 & 29.18 & 0.060 \\
\hline 17 & Leontopodium stracheyi & 20 & 0.9 & 0.023 & 3.56 & 0.045 \\
\hline 18 & Plantago lanceolata & 30 & 1.1 & 0.012 & 4.43 & 0.037 \\
\hline 19 & Pleurospermum candollei & 30 & 1.2 & 0.010 & 4.38 & 0.040 \\
\hline 20 & Poa alpina & 90 & 60.6 & 0.004 & 65.91 & 0.067 \\
\hline 21 & Poa annua & 60 & 35.5 & 0.006 & 42.14 & 0.592 \\
\hline 22 & Rheum moorcroftiasana & 20 & 0.8 & 0.366 & 16.31 & 0.040 \\
\hline 23 & Rhodiola imbricate & 20 & 0.7 & 0.342 & 15.39 & 0.035 \\
\hline 24 & Saussurea albescens & 20 & 0.7 & 0.065 & 5.07 & 0.035 \\
\hline 25 & Saussurea taraxacifolia & 20 & 0.6 & 0.231 & 11.23 & 0.030 \\
\hline \multirow[t]{2}{*}{26} & Urtica parviflora & 20 & 1.2 & 0.342 & 15.56 & 0.060 \\
\hline & Total & & 138.6 & 2.683 & & \\
\hline
\end{tabular}


of IVI (2.87). This depicts that Poa alpina was the dominant species, Poa annua codominant and Buplerium falcatum the associated species. At Dal, contagious distribution pattern of species was dominant followed by random pattern of distribution and none of the species was reported for regular distribution pattern.

Frequency, density, basal area and importance value index at Lahaud Dhar, are shown in Table 5. Jurinea dolomiaea was found to have highest frequency (70\%) followed by Gentiana kurrooa (50\%) whereas, Podophyllum hexandrum has the lowest frequency (10\%). Gentiana kurrooa has the highest density (35.8 plants $\mathrm{m}^{-2}$ ) followed by Jurinea dolomiaea (26.6 plants $\mathrm{m}^{-2}$ ) whereas, Euphorbia cognate has the lowest density (0.5 plants $\mathrm{m}^{-2}$ ). Saussurea gossypiphora has the highest basal area $\left(0.542 \mathrm{~cm}^{2}\right)$ closely followed by Jurinea dolomiaea $(0.541$ $\mathrm{cm}^{2}$ ). Gentiana kurrooa has the highest value of IVI (65.37) closely followed by Jurinea dolomiaea (65.36) whereas, Pleurospermum brunonis has the lowest value of IVI (5.54). It depicts that Gentiana kurrooa was the dominant species, Jurinea dolomiaea codominant and Pleurospermum brunonis the associated species. At Lahaud Dhar, the most of the species were distributed randomly followed by contagious pattern of distribution and the least species were reported for regular distribution pattern.

\begin{tabular}{|c|c|c|c|c|c|c|}
\hline Sr. No. & Species & $\begin{array}{l}\text { Frequency } \\
\text { (\%) }\end{array}$ & Density (plants $\mathrm{m}^{-2}$ ) & Basal area $\left(\mathrm{cm}^{2}\right)$ & IVI & $\begin{array}{l}\mathrm{A} / \mathrm{F} \\
\text { ratio }\end{array}$ \\
\hline 1 & Aconitum heterophyllum & 20 & 0.9 & 0.032 & 6.58 & 0.045 \\
\hline 2 & Angelica glauca & 30 & 1.7 & 0.041 & 9.89 & 0.057 \\
\hline 3 & Cynodon dactylon & 30 & 2.8 & 0.008 & 9.52 & 0.093 \\
\hline 4 & Euphorbia cognate & 20 & 0.5 & 0.023 & 5.79 & 0.025 \\
\hline 5 & Gentiana kurrooa & 50 & 35.8 & 0.432 & 65.37 & 0.716 \\
\hline 6 & Hypericum perphoratum & 30 & 1.2 & 0.012 & 8.11 & 0.040 \\
\hline 7 & Jurinea dolomiaea & 70 & 26.6 & 0.541 & 65.36 & 0.380 \\
\hline 8 & Onychium contigiuum & 20 & 0.8 & 0.044 & 7.01 & 0.040 \\
\hline 9 & Pedicularis carnosa & 20 & 0.7 & 0.031 & 6.33 & 0.035 \\
\hline 10 & Picrorhiza kurrooa & 30 & 1.5 & 0.028 & 9.15 & 0.050 \\
\hline 11 & Pleurospermum brunonis & 20 & 0.8 & 0.011 & 5.54 & 0.040 \\
\hline 12 & Poa alpina & 30 & 13.2 & 0.007 & 19.77 & 0.440 \\
\hline 13 & Poa annua & 20 & 12.3 & 0.005 & 16.67 & 0.615 \\
\hline 14 & Podophyllum hexandrum & 10 & 0.2 & 0.076 & 5.71 & 0.020 \\
\hline 15 & Rheum moorcroftiasana & 20 & 0.8 & 0.089 & 9.02 & 0.040 \\
\hline 16 & Saussurea gossypiphora & 20 & 0.6 & 0.542 & 29.01 & 0.030 \\
\hline \multirow[t]{2}{*}{17} & Saussurea taraxifolia & 30 & 0.5 & 0.320 & 21.17 & 0.017 \\
\hline & Total & & 100.9 & 2.242 & & \\
\hline
\end{tabular}

\section{Conclusion}

Maximum species (60) were reported from Deol area and minimum (17) from Lahaud Dhar. It has been observed that Poa alpina has the highest frequency (90\%), highest density (60.6 plants $\mathrm{m}^{-2}$ ) at Dal. Gentiana kurrooa has the highest density (35.8 plants $\mathrm{m}^{-2}$ ) at Lahaud Dhar. Moschela esculenta has the highest basal area $\left(1.234 \mathrm{~cm}^{2}\right)$ at Kut. Viola serpens was the dominant species with highest value of IVI (78.77) closely followed by Poa alpine (65.91), Gentiana kurrooa (65.37) and Jurinea dolomiaea (65.36). Most of the species were distributed randomly followed by contagious pattern of distribution and least number of species were reported showing regular distribution pattern.

\section{References}

Ahmad, R.U., 1993. Medicinal plants used in ISM-their procurement, cultivation, regeneration and import/ export aspects report. Glimpses in Plant Research. Vol. X. Medicinal Plants. New Vistas of Research. Part I (Govil, J.N., Singh, V.K. and Shamima, H., eds). Today's \& Tomorrow's Printers and Publishers, Delhi, 221-258.

Anderson, A., 1886. Report on the Demarcation and Settlement of Kullu Forests (Reprinted 1975). Himachal Pradesh Forest Deptt. Shimla.

Aryal, M., 1993. Diverted wealth: the trade in Himalayan herbs. Himal Southasian 6, 1.

Badola, H.K., 1998. Biodiversity conservation study of Kanawar 
Wildlife Sanctuary in Himachal. Research for mountain development: Some Initiatives and Accomplishments, Himavikas Publ. No. 12. Gyanodaya Prakashan, Nainital, 407-430.

Badola, H.K., 2001. Medicinal plants diversity of Himachal Pradesh. Himalayan Medicinal Plants. Potential and Prospects (Samant, S.S., Dhar, U., Palni, L.M.S., eds). Himvikas Occasional Publ. no. 14, Gyanodaya Prakashan, Nainital, 87-116.

Badola, H.K., 2002. Endangered medicinal plant speciespriorities and action. Theme Paper, Intl. workshop on Endangered Medicinal Plant Species in Himachal Pradesh. GBPIHED, MohalKullu, India (18-19 March), 11.

Chauhan, N.S., 1999. Medicinal and Aromatic plants of Himachal Pradesh. Indus Publishing Company, New Delhi, 632.

Curtis, J.T., Cottam, G., 1956. Plant Ecology Work Book Laboratory Field Reference Manual. Minnesota: Burgess Publishing Company, 193.

Curtis, J.T., Mclntosh, R.P., 1950. The interrelation of certain analytic and synthetic phytosociological characters. Ecology, 31, 434-455.

Dinanath, 2007. Studies on diversity of medicinal and aromatic plants of Pangi valley of Chamba district of Himachal Pradesh. M.Sc. Thesis. Dr. Y.S. Parmar University of Horticulture and Forestry, Nauni, Solan (H.P.) India, 223.

Dobriyal, R.M., Singh, G.S., Rao, K.S., Saxena, K.G., 1997. Medicinal plant resources in Chhakinal watershed in the North-Western Himalaya. Journal of Herbs, Spices \& Medicinal Plants 5, 15-27.

Flather, C.H., Joyce, L.A., Bloomgarden, C.A., 1994. Species endangerment patterns in the United States. USDA Forest Services General Tech. Rep. RM-241. Fort Collins, Colorado, USA, 42.

Gaur, R.D., Singh, P.B., 1993. Ethno-medicinal plants of Mandi District, Himachal Pradesh. Bulletin of Medico-Ethno Botanical Research 14, 1-14.

Gupta, R., 1964. Survey record of medicinal and aromatic plants of Chamba Forest Division of H.P. Indian forester 90, 454-468.

Gupta, R., 1971. Medicinal and aromatic plants of Bhandal Ranges, Churah Forest Division, Chamba District, Himachal Pradesh. Journal of Bombay Natural History Society $68,791-803$.
Hilton, T.C., 2000. IUCN Red list of threatened species. IUCN, Gland, Switzerland and Cambridge, U.K., 61.

Holley, J., Cherla, K., 1998. The Medicinal Plants Sector in India. Medicinal and Aromatic Plants Program in Asia (MAPPA) IDRC. SARO. IDRC, New Delhi, 91.

Karki, M.B., Willians, J.T., 1999. Priority species of medicinal plants in South Asia. New Delhi. IDRC.

Kumar, M., Joshi, M., Todaria, N.P., 2010. Regeneration status and plant bio-diversity in a sub-tropical forest of Garhwal Himalaya. Journal of Forestry Research 21, 439-444.

Lange, D., 1997. Trade figures for botanical drugs world-wide. Medicinal Plant Conservation 3, 16-17.

Lewin, R., 1986. A mass extinction without asteroids. Science, 234, 14-15.

Phillips, E.A., 1959. Methods of vegetation study. Henry Holt and Co. Inc. New York.

Raven, P.H., 1990. The politics of preserving biodiversity. Bio Science 40, 769-774.

Samant, S.S., Dhar, U., Palni, L.M.S., 1998. Medicinal Plants of Indian Himalaya: Diversity, Distribution Potential Values. Himvikas Publication No. 13, Gyanodaya Prakashan, Nainital, 163.

Shadangi, D.K., Nath, V., 2005. Imapct of seasons on ground flora under plantation and natural forest in Amarkantak. Indian Forester 131, 240-250.

Shameem, S.A., Irfana, N. Kangroo, 2011. Comparative assessment of edaphic features and phytodiversity in lower Dachigam National Park, Kashmir Himalaya, India. African Journal of Environmental Science and Technology 5, 972-984.

Tandon, V., 1997. The status of collection, Conservation, Trade and Potential for Growth in Sustainable Use of Major Medicinal Plant Species Found in the Great Himalayan National Park and its Environs in Kullu District of Himachal Pradesh. Report submitted to wildlife institute of India, Dehradun, 39.

Whitford, P.B., 1949. Distribution of woodland plants in relation to succession and clonal growth. Ecology 30, 199-208.

Wilson, E.O., 1988. The current state of biological diversity. Biodiversity (Wilson, E.O., ed.). Washington, DC: National Academy Press, 3-18. 